\title{
Archäologie der Anfänge
}

\section{Hartmut Bleumer • Mathias Herweg · Klaus Kipf}

Online publiziert: 20. Juni 2017

(C) J.B. Metzler, Part of Springer Nature, Springer-Verlag GmbH 2017

Das frühe Mittelalter ist ein symptomatischer wissenschaftsgeschichtlicher Problemfall. In seiner Erforschung zeichnen sich disziplinäre Selbstverständnisse und deren Krisen ebenso ab wie die Möglichkeiten und Grenzen des interdisziplinären Austausches. Für die Germanistik scheinen die Schwierigkeiten im Umgang mit den frühen Phasen der deutschen Sprache und Literatur besonders ausgeprägt zu sein. Nicht nur, dass die Germanistik insgesamt, aktuell prominent im Feuilleton, wieder verstärkt mit den Abgesängen auf ihr Fach zu kämpfen hat, sie hat sich auch von den eigenen Versuchen, eine Geschichte der deutschen Sprache und Literatur von ihren Anfängen her zu entwerfen, merklich distanziert und so eines ihrer elementaren Begründungsnarrative zurückgewiesen. Inzwischen zeichnet sich damit das, was man allgemein als Krise der Germanistik bezeichnen oder bestreiten mag, auch speziell im Umgang mit der frühen deutschen Sprache und Literatur erneut ab. Wenn man also den zunehmenden Bedeutungsverlust der Germanistik diagnostizieren möchte, dann muss man diese Relevanzeinbußen innerhalb der Germanistik für die Forschungen zur frühen deutschen Literatur noch einmal ansetzen.

Im interdisziplinären Vergleich zeigt sich die schleichende Marginalisierung der frühen deutschen Sprach- und Literaturgeschichte ebenfalls. Denn insbesondere die Geschichtswissenschaft, die der Germanistik ohnehin schon als produktives Ge-

\section{H. Bleumer $(\square)$}

Seminar für Deutsche Philologie, Universität Göttingen, Göttingen, Deutschland E-Mail: hbleume@gwdg.de

\section{Herweg}

Institut für Germanistik, Karlsruher Institut für Technologie, Karlsruhe, Deutschland

E-Mail: mathias.herweg@kit.edu

K. Kipf

Institut für deutsche Philologie, Ludwig-Maximilians-Universität München, München, Deutschland E-Mail: klaus.kipf@1mu.de 
genbeispiel einer öffentlichkeitswirksamen Wissenschaft vorgehalten wird, scheint selbst für das reichlich ferne Frühmittelalter keine vergleichbare disziplinäre Deflation zu kennen wie die Deutsche Philologie. Dies mag damit zusammenhängen, dass die kulturwissenschaftlichen Erweiterungen, die in den geisteswissenschaftlichen Einzeldisziplinen allenthalben zu verzeichnen sind, die Geschichtswissenschaft zu einem großen kulturwissenschaftlichen Spielfeld erweitert haben, während sie das Feld der Literaturwissenschaften auflösen. Geschichte wird gerade in ihrer je neuen Auflösung kulturell interessant, die Literatur dagegen wird flüchtig. Für die Sicht auf die Geschichte der Literatur, die in der Entwicklung der Sprache und ihrer frühen Medien beginnt, müsste das aber zweierlei bedeuten: Einerseits wäre die Betrachtung der Sprach- und Literaturzeugnisse noch stärker historisch-interdisziplinär auszurichten, andererseits wäre innerdisziplinär stärker an aktuelle methodische Entwicklungen anschließen.

Beide Forderungen wirken ziemlich wohlfeil, sind in ihren Folgen aber nicht trivial. Zum einen: So wie sich vor dem Konzept der Nation und der Nationalsprache Sprache und Literatur nicht über nationalsprachliche Einzelphilologien vermessen lassen, so erscheint auch die Geschichte insbesondere vor dem sogenannten hohen Mittelalter als ein diskontinuierlicher Pluralismus. In historischer Sicht erscheint so die Rede von der >Frühzeit< als Verlegenheitslösung gegenüber traditionellen Paradigmen, die eine historische Zukunft für eine Zeit postulieren, welche diese Zukunft noch gar nicht kennt. Darum ist im interdisziplinären Verbund der sprachliche und zeitliche Rahmen grundsätzlich offen anzusetzen, um in diesem weiten historischen Rahmen die interdisziplinären Verbindungsmöglichkeiten der Erforschung von vermeintlichen Frühphänomen auszuloten.

Zum anderen: Der erweiterte historische Rahmen erlaubt es nicht nur, spätere kulturelle oder literarische Phänomene auf ihre vermeintlichen Archaismen zu befragen. Er lässt es auch zu, Methoden- und Theorieangebote zu erproben, deren Validität für historisch spätere Phänomene schon erwiesen ist, die aber etwa für die älteste deutsche Literatur noch einer genaueren Überprüfung bedürfen. Ein solches Angebot macht für die germanistische Mediävistik aktuell insbesondere die Narratologie.

Die Beiträge des folgenden LiLi-Heftes versuchen demnach Erkundungsgänge in diese beiden Richtungen. Sie illustrieren einerseits in einem ersten Teil aus verschiedenen philologischen Perspektiven, denen der Anglistik, der Skandinavistik und der Germanistik, Möglichkeiten einer interdisziplinären Frühmittelalterforschung und ihrer möglichen Synergieeffekte. Sie unternehmen dann aber andererseits - und jenseits des engeren historischen Frühmittelalterbegriffs - diachron-narratologische Vorstöße in das weitere Feld der früheren deutschen Literatur. Dabei waren die Beiträge, die narratologische Verfahren aufgreifen, Vorträge eines Panels des Bayreuther Germanistentages 2016, das unter dem Titel >Erzählen im früheren Mittelalter. Diachron-narratologische Analysen zur deutschen Literatur vor 1170< von Klaus Kipf und Mathias Herweg konzipiert wurde. Die daraus resultierenden Aufsätze bilden daher im vorliegenden Heft einen eigenen, zweiten Abschnitt, dem die Einleitung der betreffenden Sektion zur Orientierung vorangestellt ist.

Erwähnenswert ist der 25. Germanistentag in Bayreuth als Ursprungsort dieser Vorträge schließlich auch deshalb, weil er in der Diskussion um die Krise der Germa- 
nistik als Beispiel dafür herhalten musste, im Unterschied zum wie üblich größeren und öffentlichkeitswirksameren Historikertag in Hamburg noch einmal den angeblichen Bedeutungsverlust der Germanistik zu dokumentieren. Insofern schließt sich hier ein Kreis, der dazu geeignet sein mag, ein populäres Missverständnis zu widerlegen, nämlich, dass die Relevanz einer Kulturwissenschaft von einem unmittelbar evidenten Aktualitätsbezug abhängt. In Wahrheit setzt eine solche Evidenz einen historisch weiten Weg voraus - ob in der Geschichtswissenschaft oder in den historischen Philologien. Dieses Heft der LiLi fragt in gewisser Weise auch nach den Anfängen dieses Wegs. 